\title{
LA INFLUENCIA DE LA PERSONALIDAD PARENTAL COMO PREDICTOR DE LA CONFIGURACIÓN DEL APEGO Y DE LOS PROBLEMAS DE CONDUCTA EN LA INFANCIA
}

\author{
José A. Rabadán Rubio \\ Facultad de Educación \\ Universidad de Murcia. \\ jrabadan@um.es \\ María L. Gómez Brugarolas \\ Facultad de Educación \\ Universidad de Murcia. \\ marialuisa.gomezb@um.es \\ María del Carmen Pérez Cánovas. \\ Facultad de Educación \\ Universidad de Murcia \\ mariacarmen.perezc@um.es \\ Rosario Sánchez Mazzuchelli \\ Facultad de Educación \\ Universidad de Murcia. \\ rosario.sanchezm@um.es \\ Cecilia Pérez Faz \\ Facultad de Educación \\ Universidad de Murcia. \\ cecilia.perezf@um.es
}

Fecha de Recepción: 24 Julio 2019

Fecha de Admisión: 25 Septiembre 2019

\section{RESUMEN}

El ejercicio de la paternidad se haya condicionado por los rasgos de personalidad de los progenitores (Desjardins, Zelenski \& Coplan, 2008; Lomanowska, Boívin, Hertzman \& Fleming, 2017). Las primeras huellas en la literatura científica que describen esta realidad podemos encontrarlas en el trabajo de Belsky (1984). Alude a la influencia de las características personales de los progenitores en los estilos educativos (Prinzie, Stams, Dekovic, Reijntjes \& Belsky, 2009). Vondra, Bittman y Belsky (2008) relacionan personalidad y paternidad por medio de tres dimensiones: diferenciación psicológica parental, afecto negativo y trastorno psicológico. Defienden que la habilidad para la crianza se verá modulada por la personalidad y ajuste exhibido por los progenitores. De este modo, ciertos rasgos de personalidad, tales como la psicopatía parental o emocional negativa, condicionan la apreciación de problemas conductuales en la infancia (Angelini, Klijs, Smidt \& Mierau, 2016; 


\section{LA INFLUENCIA DE LA PERSONALIDAD PARENTAL COMO PREDICTOR DE LA CONFIGURACIÓN DEL APEGO Y DE LOS PROBLEMAS DE CONDUCTA EN LA INFANCIA}

Desjardins, Zlenski \& Goplan, 2008; Lomanowka, et al., 2017; Oliver, Wright \& Coffman, 2009). De hecho, se ha encontrado que las características personales maternas predicen comportamientos infantiles. El bienestar psicológico de las madres correlaciona con los problemas en el desarrollo de sus hijos/as (Camsranesi, 2016; Nikolaev, Baranova \& Petunova, 2016). En esta investigación hemos analizado las relaciones existentes entre los rasgos de personalidad parental en el desarrollo del apego y la aparición de problemas de conducta. Para ello hemos utilizado distintas pruebas psicométricas objetivas validadas y comercializadas. A la vez, como se indica en la literatura, para la medición del apego en las edades establecidas en la investigación, hemos utilizado una prueba proyectiva. Los resultados obtenidos en el centro educativo evaluado nos ratifican y verifican una muy alta correlación en el triángulo tipo personalidad parental, configuración del apego y problemas de conducta.

Palabras clave: apego; personalidad parental; problemas de conducta.

\section{ABSTRACT}

The influence of parental personality as a predictor of the configuration of the apego and the problems of child behavior. The exercise of paternity arises from the personality traits of the parents (Desjardins, Zelenski \& Coplan, 2008; Lomanowska, Boívin, Hertzman \& Fleming, 2017). The first traces in the scientific literature that describe this reality can be found in Belsky's work (1984). It refers to the influence of the parents' personal characteristics in educational styles (Prinzie, Stams, Dekovic, Reijntjes \& Belsky, 2009). Vondra, Bittman and Belsky (2008) relate personality and paternity through three dimensions: parental psychological differentiation, negative affect and psychological disorder. They argue that the ability to raise will be modulated by the personality and adjustment exhibited by the parents. In this way, certain personality traits, such as negative parental or emotional psychopathy, condition the appreciation of behavioral problems in childhood (Angelini, Klijs, Smidt \& Mierau, 2016; Desjardins, Zlenski \& Goplan, 2008; Lomanowka, et al ., 2017; Oliver, Wright \& Coffman, 2009). In fact, it has been found that maternal personal characteristics predict child behavior. The psychological well-being of mothers correlates with the problems in the development of their children (Camsranesi, 2016; Nikolaev, Baranova \& Petunova, 2016). In this investigation we have analyzed the relationship between parental personality traits in the development of attachment and the appearance of behavioral problems. For this we have used different validated and commercialized objective psychometric tests. At the same time, as it is indicated in the literature for the measurement of attachment at the ages established in the investigation, we have used a projective test. The results obtained in the evaluated educational center ratify us and verify a very high correlation in the parental personality type triangle, attachment configuration and behavioral problems.

Keywords: attachment parental personality; behavioral problems

\section{INTRODUCCIÓN}

Bowlby $(1988,2014)$ a través de su célebre teoría del apego, establece que la personalidad y salud mental de los progenitores determina la capacidad de establecer lazos emocionales íntimos. Señala los rasgos de personalidad maternos como condicionantes para el desarrollo de un niño saludable 0 con patrones conductuales patológicos. Describe las características propias de una madre cuyo bebé se desarrolla de una manera segura: progenitoras contingentes a las necesidades de sus pequeños. Por el contrario, la madre de un bebé que exhibe un apego ansioso, es probable que muestre una esporádica asistencia al menos, respondiendo de forma tardía y/o inadecuada a las señales de niño (Abuín \& Rivera, 2015; Boonar \& Koohsar, 2011; Cameranesi, 2016). La interacción materno-filial genera en el niño "modelos operantes" de sí mismo y de los padres (Bowlby, 1988, 
2014). La vivencia que el niño/a experimenta a través de la interacción cotidiana con sus cuidadores permite al menor crearse un modelo de sí mismo, así como elaborar las imágenes que sus padres tienen de él, la forma en que prevé que lo traten y el modo que plantea se conducta hacia ellos. Por lo tanto, el tipo de apego que un niño/a desarrolla en sus primeros años de desarrollo se halla íntimamente ligado al modo en que sus padres interactúan (Abuín \& Rivera, 2015; Bonab \& Koohsar, 2011). De este modo, padres accesibles, sensibles y colaboradores permiten al niño/a confiar en sus progenitores y, por consiguiente, desarrollar un apego seguro. Esta seguridad de partida permite al niño llevar a cabo la exploración de su entorno. Por el contrario, cuando percibe que su progenitor no está accesible 0 insensible a sus necesidades, desarrolla un apego ansioso. La incertidumbre que le genera no saber si sus demandas serán atendidas le llevan a mostrarse inseguro (Ainsworth, 1971; Chen, 2017). Un tercer tipo de apego es el denominado ansioso-evitativo. Nanu y Nijloveanu (2015) describen la influencia de los tipos de apego y los subyacentes estilos educativos en el desarrollo infantil. Una implicación negligente en los padres, fundamentada en la indiferencia o una autoridad excesiva, dan como resultado un apego ansioso o evitativo en el menor. Por lo tanto, las peculiaridades en la personalidad paterna ocasionan un apego inseguro, lo que explica la evitación y ansiedad infantil, asociada a unos menores niveles de sensibilidad del cuidador (Briere, Runtz, Eadie, Bigras \& Godbout, 2017; Chen, 2017).

\section{OBJETIVOS DE LA INVESTIGACIÓN}

El objetivo general de esta investigación ha sido analizar las relaciones existentes entre los rasgos de personalidad parental en el desarrollo del apego y la aparición de problemas de conducta en la infancia. Asimismo, este objetivo general los subdividiremos en los siguientes objetivos; en primer lugar, identificar la presencia de problemas de conducta en niños/as de 3 a 6 años; en segundo lugar, conocer la anamnesis o historia clínica de los niños de 3 a 6 años que presentan problemas de conducta; en tercer lugar, valorar el tipo de apego desarrollado en los niños de 3 a 6 años que presentan problemas de conducta; en cuarto lugar, valorar el tipo de apego desarrollado en los niños de 3 a 6 años que presentan problemas de conducta, en quinto lugar, conocer los rasgos de personalidad de los padres cuyos hijos/as presentan trastornos de conducta; en sexto lugar, analizar la influencia de la personalidad parental en la aparición de diferentes patrones de apego; y, por último, analizar la influencia de la personalidad parental en la aparición de problemas de conducta.

\section{MUESTRA Y/O PARTICIPANTES}

Esta investigación forma parte de una más amplia y con una casuística muy superior y más representativa de la población de la Región de Murcia. Para esta comunicación representaremos los resultados obtenidos con la participación de niños escolarizados en $2^{\circ}$ ciclo de Educación Infantil y $1^{\circ}$ de primaria, sus familias (los cuidadores principales del niño responderán atendiendo a su rol 0 perfil - no consensuarán su respuesta, sino que se le administrarán los instrumentos de evaluación de forma independiente-) y el equipo docente (tutores/as de los niños/as) de un centro educativo público de la Región de Murcia.

\section{METODOLOGÍA Y/O INSTRUMENTOS UTILIZADOS}

Se comenzó contactando con la Consejería de Educación de la Región de Murcia para la obtención de la correspondiente autorización. Debido al perfil y situación de los centros educativos, la Consejería de Educación, de forma aleatoria, y partiendo siempre de la predisposición del centro a la participación en la investigación, se encargó de seleccionar aleatoriamente 15 centro educativos que habían, en algún momento determinado, solicitado a dicha Consejería formación y recursos por presentar dificultades o indicios de problemas de conducta en niños de infantil. Se solicitó la corres- 


\section{LA INFLUENCIA DE LA PERSONALIDAD PARENTAL COMO PREDICTOR DE LA CONFIGURACIÓN DEL APEGO Y DE LOS PROBLEMAS DE CONDUCTA EN LA INFANCIA}

pondiente autorización al Comité Ético de la Universidad de Murcia y se les remitió, con dicha autorización una carta informativa para los docentes y padres de los distintos centros. Una vez aceptada la participación, por la comunidad educativa, los tutores se encargaron de seleccionar a niños/as que presentaran los criterios de inclusión predeterminados.

Como se indica en la metodología de esta investigación, los docentes implementaron los cuestionarios SENA y SDQ de todos los niños/as preseleccionados. Una vez analizados, los alumnos que presentaban los criterios de inclusión preestablecidos se continuaban con la siguiente fase. En esta, Ios padres cumplimentaban las pruebas NEO PI-R, CUIDA y SDQ y los niños el McArthur de historias incompletas.

Las pruebas utilizadas y el objetivo de las mismas son las siguientes:

\section{Medidas de Screening}

SDQ. Strengths and Difficulties Questionnaires (Goodman, 1997).

Implementado por profesores tutores y padres del alumno evaluado.

Esta escala, con baremos actualizados gracias a los estudios de Gómez-Beneyto et al. (2013), valora marcadores clínicos conductuales relacionados con Dificultades Emocionales, Hiperactividad, Dificultades con los compañeros/as, Conducta Prosocial y Dificultades de Conducta.

\section{Medidas emocionales para padres (con referencia al niño)}

SENA. Sistema de Evaluación de Niños y Adolescentes (Sánchez, Pinto, Santamría, Ortiz y Del Barrio Gándara, 2016). Prueba implementada por los profesores con referencia a la emocionalidad del niño evaluado.

El Sistema de Evaluación de Niños y Adolescentes (SENA), valora un amplio espectro de problemas emocionales y de conducta (depresión, ansiedad, hiperactividad e impulsividad, conducta desafiante, consumo de sustancias, problemas de conducta alimentaria, dificultades de aprendizaje...), problemas contextuales (con la familia, escuela o compañeros), así como áreas de vulnerabilidad y competencia social, inteligencia emocional...).

\section{Medidas emocionales para profesores (con referencia al niño)}

SENA. Sistema de Evaluación de Niños y Adolescentes (Sánchez, Pinto, Santamría, Ortiz y Del Barrio Gándara, 2016). Prueba implementada por los profesores con referencia a la emocionalidad del niño evaluado.

El Sistema de Evaluación de Niños y Adolescentes (SENA), valora un amplio espectro de problemas emocionales y de conducta (depresión, ansiedad, hiperactividad e impulsividad, conducta desafiante, consumo de sustancias, problemas de conducta alimentaria, dificultades de aprendizaje...), problemas contextuales (con la familia, escuela o compañeros), así como áreas de vulnerabilidad y competencia social, inteligencia emocional...).

\section{Medidas de personalidad parental}

CUIDA. Cuestionario para la Evaluación de Adoptantes, Cuidadores, Tutores y Mediadores (Bermejo, Estevez, García, García-Rubio, Lapastora, Letamendía, Cruz, Polo, Sueiro y Velázquez, 2006). Implementado por padre y/o madre.

EI CUIDA ha sido creado para evaluar la capacidad de un sujeto para proporcionar la atención y el cuidado adecuados a una persona en situación de dependencia (hijo biológico, adoptado o en custodia; menor a cargo de una institución; mayores, enfermos, discapacitados...).

Incluye la evaluación de 14 variables de personalidad : Altruismo, Apertura, Asertividad, Autoestima, Capacidad de resolver problemas, Empatía, Equilibrio emocional, Independencia, 
Flexibilidad, Reflexividad, Sociabilidad, Tolerancia a la frustración, Capacidad de establecer vínculos afectivos 0 de apego, Capacidad de resolución del duelo, 3 índices de validez y control de las respuestas y 3 puntuaciones de segundo orden: Cuidado responsable, Cuidado afectivo, Sensibilidad hacia los demás y Agresividad.

NEO PI-R. Inventario de Personalidad Neo - Revisado (Costa y McCrae, 2010).

Implementado por padre y/o madre.

EI NEO PI-R es un instrumento para la evaluación de la personalidad normal. Consta de 240 elementos a los que se responde en una escala Likert de cinco opciones y permite la evaluación de cinco factores principales: Neuroticismo, Extraversión, Apertura, Amabilidad y Responsabilidad. Cada factor se descompone en seis facetas, lo que permite un análisis de la personalidad más fino, obteniendo 35 puntuaciones diferentes.

\section{Medidas de apego}

CUIDA. Cuestionario para la Evaluación de Adoptantes, Cuidadores, Tutores y Mediadores (Bermejo, Estevez, García, García-Rubio, Lapastora, Letamendía, Cruz, Polo, Sueiro y Velázquez, 2006). Implementado por padre y/o madre.

EI CUIDA ha sido creado para evaluar la capacidad de un sujeto para proporcionar la atención y el cuidado adecuados a una persona en situación de dependencia (hijo biológico, adoptado o en custodia; menor a cargo de una institución; mayores, enfermos, discapacitados...).

Incluye la evaluación de 14 variables de personalidad : Altruismo, Apertura, Asertividad, Autoestima, Capacidad de resolver problemas, Empatía, Equilibrio emocional, Independencia, Flexibilidad, Reflexividad, Sociabilidad, Tolerancia a la frustración, Capacidad de establecer vínculos afectivos o de apego, Capacidad de resolución del duelo, 3 índices de validez y control de las respuestas y 3 puntuaciones de segundo orden: Cuidado responsable, Cuidado afectivo, Sensibilidad hacia los demás y Agresividad.

MACARTHUR STORY STEM ASSESSMENT PROFILE (SSAP). Evaluación

de las representaciones mentales de apego a través de historias incompletas (Thomas MacArthur, 2003). Implementada por el niño evaluado.

Con esta prueba se valoran las expectativas y percepciones infantiles sobre las relaciones entre padres e hijos, explorando las expectativas sobre los demás como fuente de seguridad o como amenaza de insatisfacción de sus necesidades básicas, así como la representación que quien es evaluado tiene de sí mismo como persona digna de afecto y protección. Este procedimiento también permite examinar indicadores de otros aspectos importantes del funcionamiento infantil, como la regulación emocional o las relaciones con los iguales.

En este proyecto en concreto se han utilizado como herramientas para la realización del SSAP juguetes y muñecos que simulan a personas. De este modo, tanto las investigadoras como los menores nos hemos apoyado en ellos para desarrollar las historias incompletas.

A su vez, otra herramienta que nos sirvió de gran utilidad fue un "semáforo de emociones". Esta técnica es una de las más empleadas para que los menores aprendan a gestionar sus emociones. Así, mediante este recurso, los niños podían expresar sus emociones de manera más visual en el caso de que tuvieran algún tipo de impedimento para poder reflejarlas durante la prueba.

\section{RESULTADOS OBTENIDOS}

Tras la realización de la selección y cribado de los alumnos con dificultades de conducta, en el segundo ciclo de infantil, del colegio investigado, hemos obtenido una casuística de 11 sujetos de estudio. 


\section{LA INFLUENCIA DE LA PERSONALIDAD PARENTAL COMO PREDICTOR DE LA CONFIGURACIÓN DEL APEGO Y DE LOS PROBLEMAS DE CONDUCTA EN LA INFANCIA}

De los alumnos estudiados, se ha obtenido, por medio del análisis del McArthur de historias incompletas, un alumno con apego desorganizado, otro con apego evitativo y el resto con apego seguro.

Entre ellos, podemos observar, que independientemente de las dificultades manifiestas en sus conductas, una vez analizado el cuestionario SDQ implementado tanto por docentes y familiares, encontramos un importante número de comorbilidades y entre ellas fundamentalmente posibles TDAH (2), TEA (1), explosivo intermitentes (1) y la casi totalidad de ellos con inestabilidad emocional y cuadros ansiosos.

En todos ellos, podemos observar la existencia de una correlación evidente entre la inestabilidad emocional de los progenitores y las manifestaciones en las dificultades conductuales, tanto en el centro educativo, como en el entorno familiar, de los alumnos incluidos en este estudio.

\section{DISCUSIÓN Y CONCLUSIONES}

Como conclusión, señalamos que el entendimiento de las variables de personalidad y de los tipos de apego que se asociaron con las conductas problema nos va a permitir comprender el papel tan relevante que juega la familia en la transmisión de pautas de comportamiento y de socialización, tanto en el mismo entorno familiar como en el centro educativo.

Además, las evidencias mostradas nos permiten conocer elementos que nos ayudarán a planificar la intervención tanto educativa como de derivación diagnóstica, si fuera necesaria, tanto para padres como para los propios alumnos implicados en la investigación.

Por otro lado, los resultados no dejan más que una confirmación de la relevancia de la familia, ya sea por medio de los estilos educativos o por medio del aprendizaje vicario que realizan los sujetos en permanente interacción con la familia.

Finalmente, en este estudio podemos disponer de otra evidencia más con referencia a la importancia del equilibrio y estabilidad emocional de los padres con respecto a la crianza del niño.

\section{REFERENCIAS BIBLIOGRÁFICAS}

Abuín, M. R. y De Rivera, L. (2015). Dependency, detachment and psychopathology in a nonclinical simple: General relations and gender differences. Is there a new line of inquiry on paranoid pathology? Clínica y salud, 2, 65-72. doi: 10.1016/j.clysa.2015.06.003

Ainsworth, M. (1969). Object relations, dependency, and attachment: a theoretical review of the infant-mother relationship. Child Development, 4(4), 969-1025. doi: 10.111/j.14678624.1969.tb04561.x

Angelini, V., Klijs, B., Smidt, N., y Mierau, J. (2016). Associations between Childhood Parental Mental Health Difficulties and Depressive Symptoms in Late Adulthood: The Influence of LifeCourse Socioeconomic, Health and Lifestyle Factors. Plos One, 11(12). doi.org/10.1371/journal.pone. 0167703

Bayer, J. K., Ukoumunne, O.C., Mathers, M., Wake, M., Abidi, N., y Hiscock, H. (2012). Development of children's internalizing and externalizing problems from infancy to five years of age. Australian and New Zeland Journal of Psyquiatry, 46(7), 659-668.

Belsky, J. (1984). The determinants of parenting: A process model. Child Development, 55, 83-96.

Bonab, B. G., Koohsar, A, A. (2011). Relation between emotional intelligence and attachment styles in delinquent adolescents. Procedia- Social and Behavioral Sciences, 30, 963-967. Bowlby, J. (1988, 2014). Una base segura. Aplicaciones de una teoría del apego. Paidós: Psicología Profunda.

Briere, J., Runtz, M., Eadie, E., Bigras, N.,y Godbouth, N. (2017). Disengaged parenting: Structural equation modeling with child abuse, insecure attachment, and adult symptomatology. Child 


\section{PSICOLOGIA, INFANCIA Y EDUCACIÓN}

abuse \& Neglect, 67, 260-270.

Cameranesi, M. (2016). Battering typologies, attachment insecurity, and personality disorders: A comprehensive literature review. Aggression ad Violent Behavior, 28, 29-46.

Chen, B. B. (2017). Insecure attachment, resource control, and unrestricted sociosexuality: From a life history perspective. Personality and Individual Differences, 105, 213-217.

Desjardins, J., Zelenski, J. M., y Coplan, R. J. (2008). An investigation of maternal personality, parenting styles, and subjective well-being. Personality and Individual Differences, 44,(3), 587597.

Lomanowska, A. M., Boivin, M., Hertzman, C., y Fleming, A. S. (2017). Parenting begets parenting: A neurobiological perspective on early adversity and the transmission of parenting styles across generations. Neuroscience, 342, 120-139. doi: 10.1016/j.neuroscience.2015.09.029.

Nanu, E. D. y Nijloveanu, D. M. (2015). Attachment and Parenting Styles. Procedia- Social Behavioral Sciences, 203, 199-204.

Oliver, P. H., Wright, D., y Coffman, J. K. (2009). Big five parental personality traits, parenting behaviors, and adolescent behavior problems: A mediation model. Personality and Individual Differences, 47, 631-636.

Prinzie, P., Stams, J. G., Dekovie, M., Reijntjes, A. H., y Belsky, J. (2009). The

Relations Between Parents' Big Five Personality Factors and Parenting: A Meta- Analityc Review. Journal of Personality and Social Psychology, 97(2), 351-362.

Vondra, J., Sysko, H. B., \& Belsky, J. (2005). Developmental origins of parenting: Personality and relationship factors. In T. Luster \& L. Okagaki (Eds). Parenting: An ecological perspective $\left(2^{\text {nd }}\right.$ ed., pp 35-71). Mahwah, N. J.: Lawrence Erlbaum. 
